\title{
Photoluminescence of the GaAs Superlattices with Quasi-Delta-Doped Layers
} \author{
and D.V. Ushakov

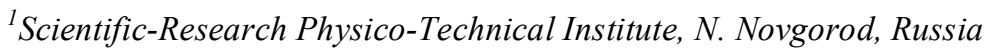 \\ ${ }^{2}$ Institute for Physics of Microstructures RAS, N. Novgorod, Russia \\ ${ }^{3}$ Stepanov Institute of Physics NASB, Minsk, Belarus \\ ${ }^{4}$ University of Pretoria, Pretoria, South Africa \\ ${ }^{5}$ Belarussian State University, Minsk, Belarus
}

B.N. Zvonkov ${ }^{1}$, V.Ya. Aleshkin ${ }^{2}$, V.I. Gavrilenko ${ }^{2}$, V.K. Kononenko ${ }^{*}, 3$, H.W. Kunert ${ }^{4}$, S.V. Morozov ${ }^{2}$

\begin{abstract}
The GaAs short-period superlattices have been grown for the first time by the metal-organic hydride epitaxy method using Se and $\mathrm{C}$ for quasi- $\delta$-doping. Photoluminescence spectra measured at $4.2 \mathrm{~K}$ display well-distinguished peaks, which coincide with transitions between quantizied levels of electrons and holes in the potential relief quantum wells. To describe observed phenomena in quasi- $\delta$-doped superlattices a theory is developed with taking into account existing tails of the density of states.
\end{abstract}

Keywords: GaAs, superlattice, $\delta$-doping, quantum well, photoluminescence, tunability.

\section{INTRODUCTION}

Doping superlattices, or $n-i-p-i$ crystals, have advanced properties for the development of novel optoelectronic devices. The $\delta$-doping technology extends possibilities of the superlattice design and allows improving the characteristics compared with homogeneously doped $n-i-p-i$ structures. For the $\delta$-doping, molecular-beam epitaxy is most widely applied [1]. The GaAs $\delta$-doped superlattices were also grown by metal-organic vapor-phase epitaxy [2, 3]. For $n$ - and $p$-type $\delta$-doping, impurities $\mathrm{Si}$ and $\mathrm{C}$ were used and for obtained superlattices the tunable low-temperature photoluminescence (PL) was demonstrated.

Tunable $n-i-p-i$ structures are attractive for integration with different optoelectronic devices, such as photoelectric detectors [4], quantum cascade lasers [5], or photonic crystal heterostructures [6]. Besides, $\delta$-doped superlattices provide effective operation parameters and extent functional characteristics of photodetectors [7], modulators [8-10], and nonlinear optical switches [11-13].

In this work, the GaAs short-period superlattices have been grown for the first time by the metal-organic hydride epitaxy method using Se and C for quasi- $\delta$-doping. Earlier, such a method was applied for making the photosensitive heterostructures with the GaInAs quantum-well spacer [14]. For the obtained superlattices, low-temperature PL spectra were measured and a theory for describing the observed phenomena was developed.

*Address correspondence to this author at the Stepanov Institute of Physics NASB, Independence Ave., 68, 220072 Minsk, Belarus; Tel: +375 17284 04 35; Fax: +375 1728408 79; E-mail: lavik@dragon.bas-net.by

\section{EXPERIMENTAL}

The formed GaAs quasi- $\delta$-doped superlattices have the 30 -period $n-i-p-i$ set of doped $n$ - and $p$-type layers and undoped $i$-layers. The superlattice period $d$ was 19 or $29 \mathrm{~nm}$ and $i$-layers were, accordingly, of the order of 7 or $11 \mathrm{~nm}$. The structure thickness was 0.8 or $1.2 \mu \mathrm{m}$, including the 0.2 $\mu \mathrm{m}$ buffer layer and $0.1-\mu \mathrm{m}$ top layer. The sheet concentrations of donors Se in the $n$-layers and of acceptors $\mathrm{C}$ in the $p$-layers made up $(1.5-1.6) \times 10^{13} \mathrm{~cm}^{-2}$.

The PL characteristics of the grown $\delta$-doped superlattices were measured at $\mathrm{CW}$ excitation by the $488-\mathrm{nm}$ radiation of an $\mathrm{Ar}^{+}$-ion laser. At the structure temperature $T=77 \mathrm{~K}$ in the spectral region near $1.4 \mathrm{eV}$, a tunable PL band in dependence on power excitation is observed [15]. The PL spectrum for the longer-period structures is shifted to the long-wavelength region and its tuning bandwidth is larger as compared the shorter-period structures. Since the $i$-layers in the first structures is approximately in one and a half times thicker than for another structures, the superlattice effective energy band gap must be smaller for the first ones and it exhibits a greater tunability under optical excitation. In addition, it provides a narrower linewidth of the PL spectrum.

The PL spectra measured at $4.2 \mathrm{~K}$ (Fig. 1) display a structure shape with well-distinguished peaks, which coincide with allowed optical transitions between quantizied levels of electrons and holes in the potential relief quantum wells. Such a behavior has been also observed for structures grown by gas-source molecular-beam epitaxy [16].

\section{THEORY}

To describe observed PL phenomena in quasi- $\delta$-doped superlattices, we used the simulation procedure previously 
developed and successfully applied for the description of PL spectra of homogeneously and $\delta$-doped superlattices as well $[17,18]$. The theory with taking into account existing tails of the density of states explains adequately experimental observations of the tuning and transformation of PL spectra and shows that at increasing the temperature the quantized character of the spectra is diminished.

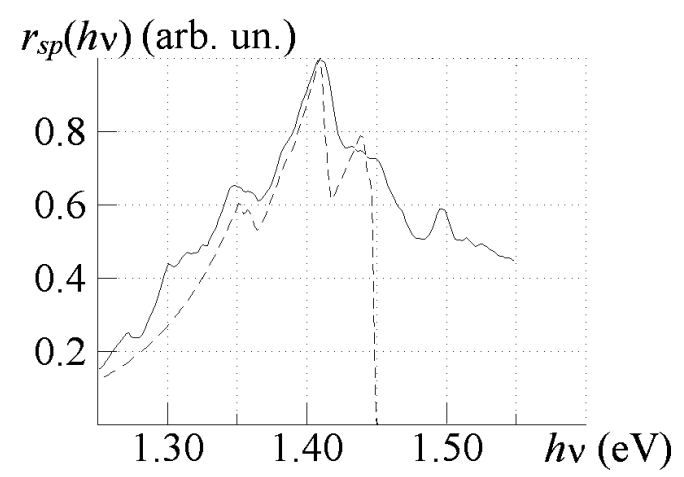

Fig. (1). The PL spectrum $r_{\text {sp }}(h v)$ of the quasi- $\delta$-doped GaAs $n-i-p-i$ structure at $T=4.2 \mathrm{~K}$ as compared a calculated spontaneous emission recombination spectrum (the dashed curve) at $\Delta F=1.472 \mathrm{eV}$.

As seen from Fig. (1), the best fitting between experimental and calculated PL spectra occurs where the superlattice design parameters are assumed to be as followed, i. e., acceptor concentration $N_{\mathrm{a}}=5.17 \times 10^{19} \mathrm{~cm}^{-3}$, donor concentration $N_{\mathrm{d}}=1.5 \times 10^{20} \mathrm{~cm}^{-3}$, $p$-layer thickness $d_{p}$ $=2.9 \mathrm{~nm}, n$-layer thickness $d_{n}=1 \mathrm{~nm}, i$-layer thickness $d_{i}=5.55 \mathrm{~nm}$, number of periods $N_{p}=10$, effective electron mass $m_{\mathrm{c}}=0.067 m_{\mathrm{e}}$, effective heavy hole mass $m_{\mathrm{vh}}=0.34 m_{\mathrm{e}}$, effective light hole mass $m_{\mathrm{vl}}=0.094 m_{\mathrm{e}}$, transverse heavy hole mass $m_{\mathrm{vh} \perp}=0.11 m_{\mathrm{e}}$, transverse light hole mass $m_{\mathrm{vl} \perp}=0.20 m_{\mathrm{e}}$. If a superlattice is excited up to the value of the quasi-Fermi level difference $\Delta F$ of $\simeq 1.47 \mathrm{eV}$ at $T=4.2 \mathrm{~K}$, the effective energy gap $E_{\mathrm{g}}^{\prime}$ equals $\simeq 1.04 \mathrm{eV}$ and the potential profile depth $2 \Delta V \simeq 0.45 \mathrm{eV}$. In this case, distribution of quantized electron and hole levels in the periodic quantum wells is shown in Fig. (2).

Obviously, not only the population of the excited subband states determines the intensity and shape of the emission band of the structures but also the strength of overlapping the electron and hole envelope wave functions is essential. Comparison of the observed PL spectrum and the numerical simulated shape of the recombination emission band shows that more intensive transitions occur through the ground subband of electrons (the quantum number $n=0$ ) and light hole states with quantum numbers $m=0,1$. At high enough excitation, significant value has transitions involving excited electron states $(n=1)$ and ground subbands of heavy and light holes $(m=0)$. For the optical transitions through the light hole subband, the squired overlap integral of the electron and hole envelope wave functions $\left|I_{n m}\right|^{2}$ has the highest value (see Table 1).

Character of a tunability of the PL band and transformation of its shape under excitation of the superlattice at different temperatures are shown in Fig. (3). Increasing the temperature results in a smooth of the PL band. The tuning bandwidth reaches up to $0.4 \mathrm{eV}$.

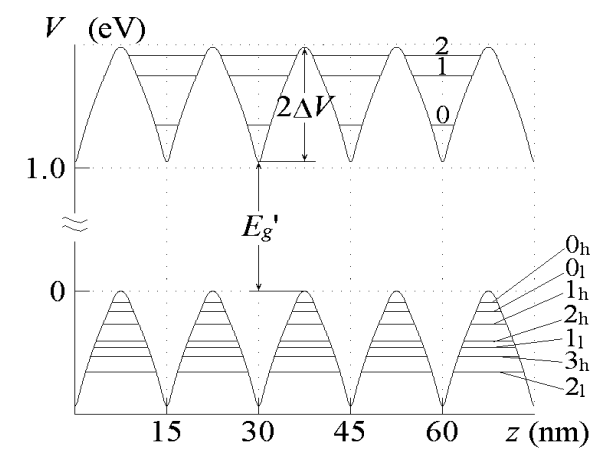

Fig. (2). Potential profile $V(z)$ of the modeling $n-i-p-i$ structure and energy levels of electrons and heavy (h) and light (l) holes. $T=4.2 \mathrm{~K}, \Delta F=1.472 \mathrm{eV}$.

However, it has to mention that at low excitation power the intensity of the tunable PL band markedly decreases and a broad emission band in the range of $1 \mathrm{eV}$ masks it. The observed additional broad PL band can be attributed to deep levels of residual impurities or related complexes [15]. Available peaks at the short-wavelength edge of the tunable PL band are evidently related to carbon, exciton recombination, and direct band-band transitions.

Table 1. Energies of Optical Transitions $h v_{n m}$ Between Electron $\left(E_{\mathrm{c} n}\right)$ and Heavy (h) or Light (I) Hole $\left(E_{\mathrm{v} m}\right)$ Energy States with Different Quantum Numbers $n$ and $m$ and the Miniband Wave Vector $k_{z}=0, \pi / d$ and Values of the Squared Overlap Integral of the Electron and Hole Envelop Wave Functions $\left|I_{n m}\right|^{2}$

\begin{tabular}{|c|c|c|c|c|}
\hline $\boldsymbol{h} \boldsymbol{v}_{n \boldsymbol{m}}(\mathrm{eV})$ & $\left(\boldsymbol{n}, \boldsymbol{m}, \boldsymbol{k}_{\mathbf{z}}\right)$ & $\boldsymbol{E}_{\mathrm{c} n}(\mathrm{eV})$ & $\boldsymbol{E}_{\mathrm{v} \boldsymbol{m}}(\mathbf{e V})$ & $\left|\boldsymbol{I}_{\boldsymbol{n} \boldsymbol{m}}\right|^{\mathbf{2}}$ \\
\hline \hline 1.221 & $(0,0 \mathrm{~h}, 0)$ & 0.134 & 0.047 & 0.011 \\
\hline 1.258 & $(0,01,0)$ & 0.134 & 0.084 & 0.048 \\
\hline 1.309 & $(0,1 \mathrm{~h}, \pi / \mathrm{d})$ & 0.135 & 0.134 & 0.038 \\
\hline 1.379 & $(0,2 \mathrm{~h}, 0)$ & 0.134 & 0.205 & 0.109 \\
\hline 1.402 & $(0,11, \pi / \mathrm{d})$ & 0.135 & 0.227 & 0.245 \\
\hline 1.407 & $(1,0 \mathrm{~h}, \pi / \mathrm{d})$ & 0.319 & 0.048 & 0.207 \\
\hline 1.442 & $(0,3 \mathrm{~h}, \pi / \mathrm{d})$ & 0.135 & 0.267 & 0.216 \\
\hline 1.443 & $(1,01, \pi / \mathrm{d})$ & 0.319 & 0.084 & 0.388 \\
\hline
\end{tabular}

\section{ANALYTICAL APPROACH}

Qualitative analysis can be provided in the frame of the sawtooth superlattice model [16, 19-21]. Analytical simulation of the redistribution of the energy subbands and, accordingly, of the optical transition quantum energies for a superlattice excited at low temperatures approves and supports conclusions based on the above described more complex numerical calculations.

In the effective impurity concentration approach, the potential relief depth $2 \Delta V$ of a quasi- $\delta$-doped superlattice is described by the relation [17]:

$$
\begin{aligned}
2 \Delta V & =2 \Delta V_{0}(1-r) \\
& =\frac{\pi e^{2}}{\varepsilon} \frac{N_{\delta}}{2}\left(d+2 d_{i}\right)(1-r),
\end{aligned}
$$


where $\varepsilon$ is the dielectric constant, $N_{\delta}=N_{\mathrm{d}} d_{n}=N_{\mathrm{a}} d_{p}, r=n / N_{\delta}$ is the excitation factor, $n$ is the non-equilibrium concentration of electrons. The energy of quantizied levels depends also on the superlattice excitation power. For a sawtooth potential relief, the level energies can be calculated with the high enough accuracy using the effective mass approximation and transforming the Schrödinger equation into the Airy differential equation [19-21]. The Airy function asymptotic expansion approach [19] and variational calculations [16] provide almost the same results for the lowest several confined states. For the electron states $E_{\mathrm{c} n}$ we have analytically:

$$
\begin{aligned}
E_{\mathrm{c} n} & =\left(\frac{3 \pi}{4}\right)^{2 / 3}\left(n+\frac{1}{2}\right)^{2 / 3}\left(\frac{\pi e^{2}}{\varepsilon}\right)^{2 / 3}\left(\frac{2 \hbar^{2} N_{\delta}^{2}}{m_{\mathrm{c}}}\right)^{1 / 3} \\
& \times(1-r)^{2 / 3}=E_{00}(2 n+1)^{2 / 3}(1-r)^{2 / 3},
\end{aligned}
$$

where the quantum number $n=0,1,2, \ldots, E_{00}$ is the ground electron state energy. Here, levels of the appeared minibands are not included, their width is assumed to be negligible small. Similar expressions are given for heavy and light hole states $E_{\mathrm{v} m}$.

(a)

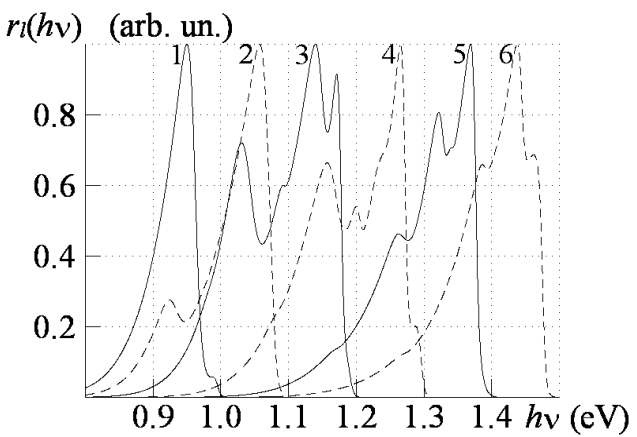

(b)

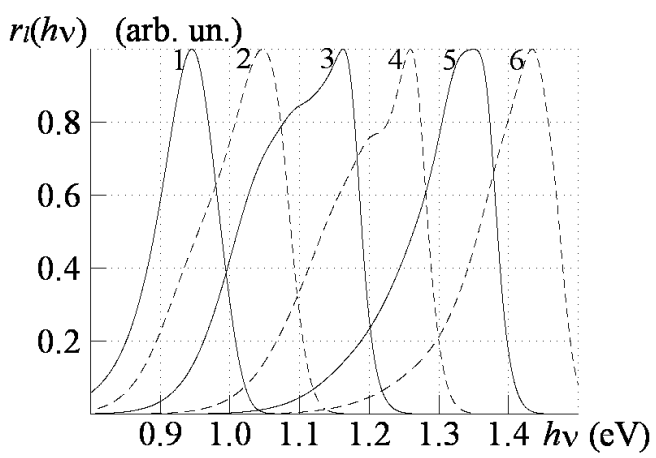

Fig. (3). Transformation of the PL spectrum $r_{1}(h v)$ of the $n-i-p-i$ structure at different temperatures (a) $T=20$ and (b) $77 \mathrm{~K}$ versus the quasi-Fermi level difference (1) $\Delta F=1.0$, (2) 1.1 , (3) 1.2 , (4) $1.3,(5) 1.4$, and (6) $1.5 \mathrm{eV}$.

Thus, we obtain a simple expression for determining the energy $h v_{n m}$ of emitted quanta, which are associated with transitions between electron states $E_{\mathrm{c} n}$ and hole states $E_{\mathrm{v} m}$, i.e.,

$$
\begin{aligned}
h v_{n m} & =E_{\mathrm{g}}-2 \Delta V_{0}(1-r)+E_{00}(1-r)^{2 / 3} \\
& \times\left((2 n+1)^{2 / 3}+\left(\frac{m_{\mathrm{c}}}{m_{\mathrm{v} i}}\right)^{1 / 3}(2 m+1)^{2 / 3}\right),
\end{aligned}
$$

where $E_{\mathrm{g}}$ is the energy gap of the semiconductor, $i=\mathrm{h}, 1$ is the hole index. As seen, at increasing the superlattice excitation the quantum energy $h v_{n m}$ approaches $E_{\mathrm{g}}$ since the potential relief depth decreases, and the energies of various optical transitions become closer as a result of decreasing the energy levels, which are involved in the transitions.

To find relation between the quasi-Fermi levels $F_{\mathrm{e}}$ and $F_{\mathrm{h}}$ and to evaluate the excitation factor $r$, we use the electroneutrality equation for degenerate conditions that are accomplished at low temperatures, i.e.,

$$
\begin{aligned}
& m_{\mathrm{c}} \sum_{n}\left(F_{\mathrm{e}}-E_{\mathrm{c} 0}-E_{\mathrm{c} n}\right) \\
& =\sum_{m, i} m_{\mathrm{v} i \perp}\left(E_{\mathrm{v} 0}-E_{\mathrm{v} m}-F_{\mathrm{h}}\right),
\end{aligned}
$$

where $E_{\mathrm{c} 0}-E_{\mathrm{v} 0}=E_{\mathrm{g}}^{\prime}, F_{\mathrm{e}}-E_{\mathrm{c} 0} \geq E_{\mathrm{c} n}, E_{\mathrm{v} 0}-F_{\mathrm{h}} \geq E_{\mathrm{v} m}$. In the parabolic band approximation, transverse components of effective hole masses are taken as $m_{\mathrm{v} i \perp}=m_{\mathrm{v} i}$. Calculations are carried out with the following parameters $N_{\delta}=1.5 \times 10^{13} \mathrm{~cm}^{-2}$, $d=19 \mathrm{~nm}, d_{i}=6.8 \mathrm{~nm}, m_{\mathrm{c}}=0.067 m_{\mathrm{e}}, m_{\mathrm{vh}}=0.34 m_{\mathrm{e}}, m_{\mathrm{vl}}=$ $0.094 m_{\mathrm{e}}, E_{\mathrm{g}}=1.519 \mathrm{eV}, \varepsilon=12.5$. In this case, the initial depth of the potential profile equals $2 \Delta V_{0}=0.885 \mathrm{eV}$.

At increasing the excitation of the superlattice, $e$. g., up to $r$ $=0.45$ and $\Delta F=1.5 \mathrm{eV}$, the relief depth becomes $2 \Delta V=0.487$ $\mathrm{eV}$ and current carriers occupy ground and excited electron subbands and only ground heavy and light hole subbands. Therewith, the effective energy gap $E_{\mathrm{g}}^{\prime}=1.032 \mathrm{eV}$ and a set of allowed optical transitions includes quantum energies 1.256, $1.300,1.408$, and $1.452 \mathrm{eV}$. The energies 1.256 and $1.300 \mathrm{eV}$ are attributed to transitions between the ground electron $(n=0)$ and heavy and light hole $(m=0)$ subbands, and the energies 1.408 and $1.452 \mathrm{eV}$ are associated with transitions from the excited electron states $(n=1)$ to the ground $(m=0)$ heavy and light hole subbands, respectively. It is in accordance with data [16] and numerical more precise self-consistent calculations (see Table 1).

\section{CONCLUSION}

Spectra of PL emission measured at $4.2 \mathrm{~K}$ display a structure shape with well-distinguished peaks, which coincide with allowed optical transitions between quantizied levels of electrons and holes in the potential relief quantum wells. The developed theory with taking into account tails of the density of states explains experimental observations and shows that at increasing the temperature the quantized spectra behavior becomes less pronounced. The main attribute of doping superlattices, $i$. e., tunability of the emission spectrum in dependence on the excitation power is observed in a wide range 1.3 to $1.5 \mathrm{eV}$ at the structure temperature up to $77 \mathrm{~K}$. Tunable performance of the $n-i-p-i$ structures is important for extension of functional characteristics of various optoelectronic devices, including 
photodetectors, modulators, amplifiers, lasers, light-emitting diodes, and nonlinear optical switches.

\section{REFERENCES}

[1] Schubert EF. Optical properties of $\delta$-doped doping superlattices. Surf Sci 1990; 228: 240-6.

[2] Li G, Jagadish C, Johnston MB, Gal M. Growth of Si and C $\delta$ doped nipi doping superlattices in GaAs by metal organic vapor phase epitaxy. Appl Phys Lett 1996; 69: 4218-20.

[3] Johnston MB, Gal M, Li G, Jagadish C. Photoluminescence study of the dynamical properties of GaAs sawtooth superlattices. J Appl Phys 1997; 82: 5748-52.

[4] Ulrich B, Zhang C, Klitzing K. Quantum-confined subband transitions of a GaAs sawtooth doping superlattice. Appl Phys Lett 1989; 54: 1133-5.

[5] Faist J, Müller A, Beck M, et al. A quantum cascade laser based on an $n-i-p-i$ superlattice. IEEE Photon Technol Lett 2000; 12: 263-5.

[6] Smirnov AG, Ushakov DV, Kononenko VK. Multiple-wavelength lasing in one-dimensional band gap structures: implementation with active $n-i-p-i$ layers. J Opt Soc Am B 2002; 19: 2208-14.

[7] Osipov VV, Selyakov AYu, Foygel M. Interband absorption of long-wavelength radiation in $\delta$-doped superlattices based on singlecrystal wide-gap semiconductors. Semicond 1998; 32: 201-5.

[8] Pfeiffer U, Kneissl M, Knüpfer B, et al. Dynamical switching behavior of $n-i-p-i$ modulator structures. Appl Phys Lett 1996; 68: 1838-40.

[9] Beck M, Streb D, Vitzethum M, et al. Ambipolar drift of spatially separated electrons and holes. Phys Rev B 2001; 64: 085307-1-8.

[10] Tribuzy CV-B, Landi SM, Pires MP, et al. nipi Delta-doping superlattices for amplitude modulation. Brazilian J Phys 2002; 32: 269-74.

[11] Kost AR, Jupina MH, Hasenberg TC, Garmire EM. Wavelength dependence of combined local and carrier transport optical nonlinearities in a hetero $n-i-p-i$ structure. J Appl Phys 2006; 99: 023501-1-4.

[12] Saidi I, Bouzaïene L, Maaref H, Mejri H. Optical nonlinearities in delta-doped $\mathrm{AlGaN} / \mathrm{GaN}$ quantum well heterostructures. J Appl Phys 2007; 101: 094506-1-6.

[13] Ushakov DV, Kononenko VK, Marciniak M. Nonlinearities in the reflection and transmission spectra of the photonic bandgap heterostructures with $n-i-p-i$ crystals. Opt Quant Electron 2007; 39: 491-9.

[14] Karpovich A, Aleshkin VYa, Anshon AV, Babushkin TS, Zvonkov BN, Malkina IG. Photoelectric properties of epitaxial GaAs/InGaAs quantum-well heterostructures. Sov Phys Semicond 1990; 24: 1346-8.

[15] Zvonkov BN, Aleshkin VYa, Gavrilenko VI, et al. Optical characteristics of the GaAs delta-doped superlattices. Bull Russian Acad Sci Ser Phys 2004; 68(1): 84-6.

[16] Schubert EF, Harris TD, Cunningham JE, Jan W. Multisubband photoluminescence in sawtooth doping superlattices. Phys Rev B 1989; 39: 11011-5.

[17] Kononenko VK, Manak IS, Ushakov DV. Optoelectronic properties and characteristics of doping superlattices. In: Proceedings of Conferences \& Publications on Photonics, Optics, \& Imaging 1998; vol. 3580: pp. 10-27.

[18] Ushakov DV, Kononenko VK, Manak IS. Effects of energyspectrum broadening in alloyed semiconductor superlattices. J Appl Spectrose 1999; 66: 820-5.

[19] Schubert EF, Ulrich B, Harris TD, Cunningham JE. Quantumconfined interband absorption in GaAs sawtooth-doping superlattices. Phys Rev B 1988; 38: 8305-8.

[20] Ferreyra JM, Proetto CR. Electronic band structure of GaAs sawtooth-doping superlattice. Phys Rev B 1990; 42: 565764.

[21] Ahn D. Intersubband transitions in a $\delta$-doped semiconductor with an applied electric field: Exact solutions. Phys Rev B 1993; 48: 7981-5.

(C) Zvonkov et al.; Licensee Bentham Open

This is an open access article licensed under the terms of the Creative Commons Attribution Non-Commercial License (http://creativecommons.org/licenses/by$\mathrm{nc} / 3.0 /$ ) which permits unrestricted, non-commercial use, distribution and reproduction in any medium, provided the work is properly cited. 